\title{
Encouraged 6-min Walking Test Indicates Maximum Sustainable Exercise in COPD Patients*
}

\author{
Alejandro Casas, MD; Jordi Vilaro, PT; Roberto Rabinovich, MD; \\ Anamaria Mayer, PT; Joan Albert Barberà, MD; \\ Robert Rodriguez-Roisin, MD, FCCP; and Josep Roca, MD
}

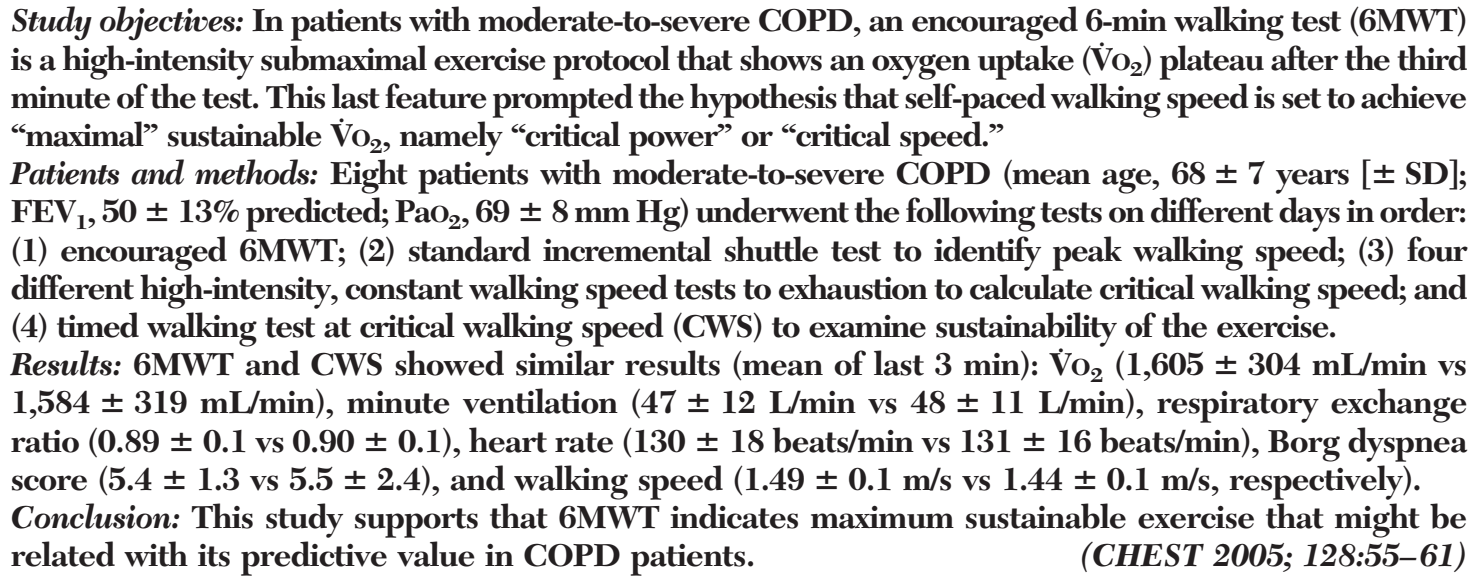

Key words: COPD; critical power; exercise testing; exercise tolerance; 6-min walking test; sustainable submaximal exercise

Abbreviations: $\mathrm{ANOVA}=$ analysis of variance; $6 \mathrm{MWT}=6$-min walking test; $6 \mathrm{MWT}-10=6$-min walking test carried out in a 10-m course; 6MWT-90 = 6-min walking test carried out in a 90-m course; CWS = critical walking speed; GOLD = Global Initiative for Chronic Obstructive Lung Disease; $\mathrm{HR}=$ heart rate; $\mathrm{L}_{1}=90 \%$ of the peak walking speed; $\mathrm{L}_{2}=95 \%$ of the peak walking speed; $\mathrm{L}_{3}=100 \%$ of the peak walking speed; $\mathrm{L}_{4}=105 \%$ of the peak walking speed; RER = respiratory exchange ratio; $\dot{\mathrm{V}_{\mathrm{CO}}}=$ carbon dioxide output; $\dot{\mathrm{VE}}=$ minute ventilation; $\dot{\mathrm{VO}}_{2}=$ oxygen uptake; $\dot{\mathrm{V}}_{2}$ peak = peak oxygen uptake

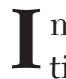
mpairment of exercise tolerance in COPD patients has important implications on health-related quality of life, ${ }^{1-3}$ hospitalization rate, ${ }^{4,5}$ and

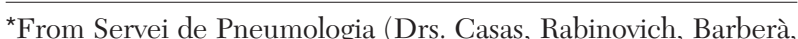
Rodriguez-Roisin, and Roca, and Ms. Mayer), Hospital Clínic, IDIBAPS, Universitat de Barcelona; and EUIF Blanquerna (Dr. Vilaro), Universitat Ramon Llull, Barcelona, Spain.

Alejandro Casas was a Pre-doctoral Research Fellow from Fundación Neumológica Colombiana (Colombia) supported by CHRONIC (IST-1999/12158) from the European Union; and, Anamaria Mayer was a Research Fellow from Federal University of Sao Paulo (Brasil) supported by CAPES and E-Remedy (IST-2000/25146).

Supported by grants FIS 00/0281 from the Fondo de Investigaciones Sanitarias; and Comissionat per a Universitats i Recerca de la Generalitat de Catalunya (1999 SGR 00228); and Red Respira C03/11.

Manuscript received November 14, 2003; revision accepted January 4, 2005.

Reproduction of this article is prohibited without written permission from the American College of Chest Physicians (www.chestjournal. org/misc/reprints.shtml).

Correspondence to: Josep Roca, MD, Servei de Pneumologia, Hospital Clínic, Villarroel 170, 08036 Barcelona, Spain; e-mail: jroca@clinic.ub.es survival. ${ }^{6,7}$ Consequently, exercise testing is progressively being considered an essential component in the routine clinical assessment of functional status.

Recommendations on exercise testing in lung function laboratory settings are well established, ${ }^{8-10}$ and the role of incremental cardiopulmonary cycling exercise as the "gold standard" for evaluation of exercise tolerance is acknowledged. We are facing, however, a widespread clinical use of simple exercise protocols $^{3,11,12}$ because of their simplicity, applicability, and low cost. Moreover, timed walking tests have been shown to predict survival ${ }^{6}$ and utilization of health-care resources ${ }^{4,5}$ in COPD patients. Therefore, it is suggested that simple exercise tests may be useful for staging of the disease. ${ }^{7}$

In a study ${ }^{13}$ conducted in patients with moderateto-severe COPD, we reported the 6 -min walking test (6MWT) as a high-intensity. submaximal exercise protocol that shows an exponential oxygen uptake $\left(\mathrm{V}_{2}\right)$ increase up to a plateau during the last $3 \mathrm{~min}$ 
of the test. From these results, we hypothesized that the self-paced walking speed during the test is set to achieve "maximal" sustainable exercise, which, by definition, corresponds to patient's critical power, ${ }^{14}$ or critical walking speed (CWS), defined as the maximum sustainable walking speed.

In the current study, we estimated the CWS in eight patients with moderate-to-severe COPD. The physiologic responses during the 6MWT were compared with those obtained during walking at CWS in the same patients. Identity between 6MWT and CWS may help to explain the high predictive value of 6MWT in chronic disorders. ${ }^{15,16}$ An ancillary purpose of the investigation was the analysis of $\dot{\mathrm{V}}_{2}$ profiles in four different common clinical exercise protocols performed in the same patients: incremental cycling exercise, 6MWT, incremental shuttle test, and stair-climbing test. ${ }^{10}$

\section{Materials AND Methods}

Eight men with clinically stable COPD were recruited for the study. All of the men had a lack of exacerbations in the preceding 6 weeks. Oxyhemoglobin desaturation during exercise was an exclusion criteria. All patients were receiving inhaled, long-acting $\beta_{2}$-agonists and ipratropium bromide. Five of patients were regularly treated with inhaled steroids, but none of the eight patients had received systemic steroids in the last 3 months prior to the study. The study design included two phases: (1) comparison between 6MWT and walking at CWS, and (2) analysis of physiologic responses during the four different clinical exercise protocols.

\section{Comparison Between 6MWT and Walking at CWS}

All patients performed, in sequence, the following timed walking tests carried out in a $10-\mathrm{m}$ course to allow comparability among exercise protocols: (1) encouraged 6MWT (6MWT carried out in a 10-m course [6MWT-10]); (2) standard incremental shuttle test; (3) four high-intensity timed-walking tests at different constant speeds to calculate CWS (Fig 1), done in random order on separate days; and (4) an additional timed walking test at CWS (Fig 1), carried out to demonstrate the sustainability of the effort at least for $20 \mathrm{~min}$ and to compare with the physiologic responses during 6MWT-10.

\section{Calculation of CWS}

The four different high-intensity, constant-walking, speed exercises (Fig 1) were specifically chosen at approximately $90 \%$ of the peak walking speed $\left(\mathrm{L}_{1}\right), 95 \%$ of the peak walking speed $\left(\mathrm{L}_{2}\right)$, $100 \%$ of the peak walking speed $\left(\mathrm{L}_{3}\right)$, and $105 \%$ of the peak walking speed $\left(\mathrm{L}_{4}\right)$ obtained during the incremental shuttle test. The CWS for a given patient corresponds to the asymptote of the hyperbolic function defined by walking speed and time to exhaustion $^{17-20}$ (Fig 1, center, B). This hyperbolic function is mathematically transformed into a linear relationship when the reciprocal of time to exhaustion (1/time) is used in the $\mathrm{x}$-axis (Fig 1 , bottom, C). CWS is defined by the $y$-intercept obtained using the equation proposed by Neder et al. ${ }^{14}$
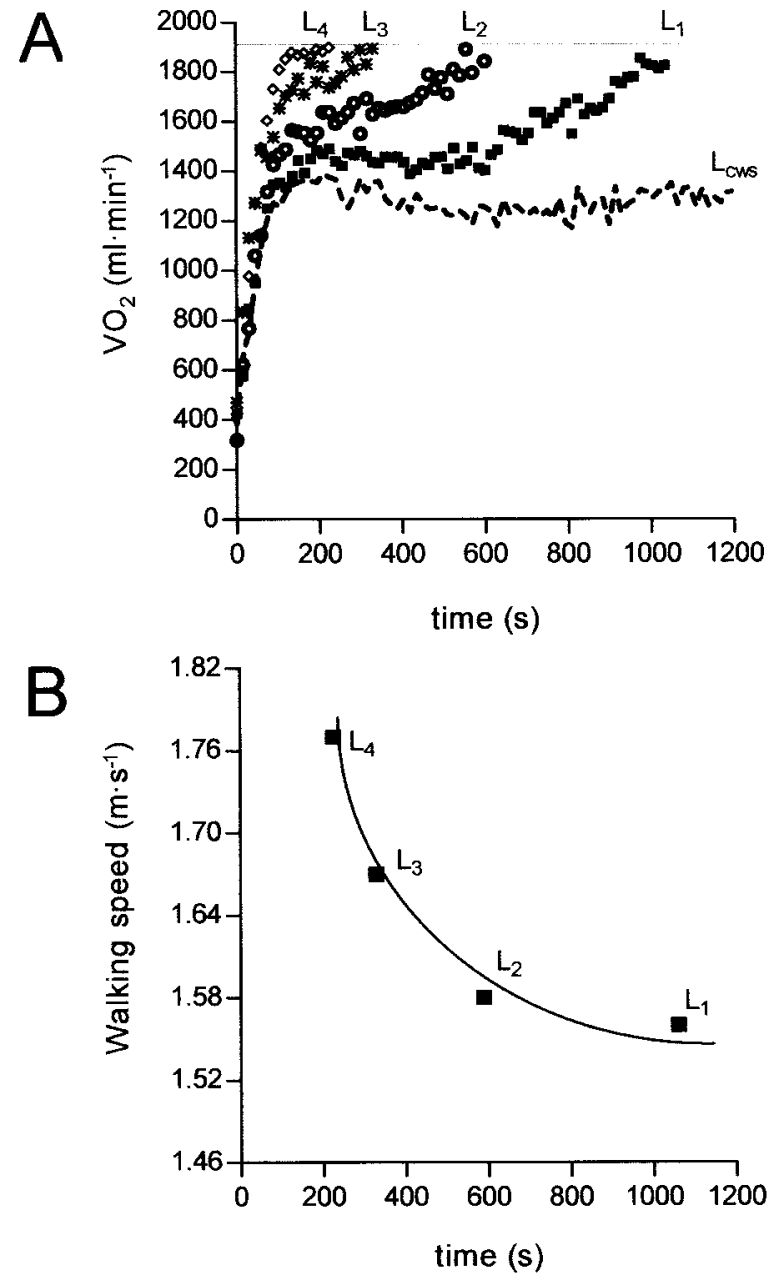

C

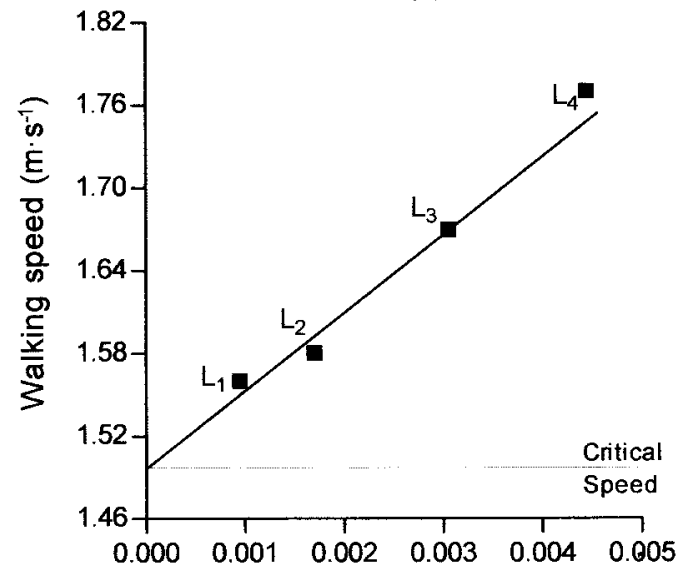

$1 /$ time $\left(s^{-1}\right)$

Figure 1. Calculation of CWS in a representative patient. Top, A: $\dot{\mathrm{VO}}_{2}$ profiles in four high-intensity tests at constant walking speed $\left(\mathrm{L}_{1}, 1.56\right.$ $\mathrm{m} / \mathrm{s} ; \mathrm{L}_{2}, 1.58 \mathrm{~m} / \mathrm{s} ; \mathrm{L}_{3}, 1.67 \mathrm{~m} / \mathrm{s}$; and $\mathrm{L}_{4}, 1.75 \mathrm{~m} / \mathrm{s}$, which correspond to approximately $90 \%, 95 \%, 100 \%$, and $105 \%$ of the peak walking speed during a standard incremental shuttle test, respectively). In three of the four tests, maximum whole-body $\mathrm{VO}_{2}$ was reached at exhaustion. The fifth constant walking speed test was done at CWS (LCWs, $1.50 \mathrm{~m} / \mathrm{s}$ ). Center, B: The asymptote of the hyperbolic relationship between walking speed (y-axis) and time to exhaustion $\left(\mathrm{L}_{1}\right.$ to $\mathrm{L}_{4}$ respectively, $1,060 \mathrm{~s}, 589 \mathrm{~s}, 328 \mathrm{~s}$, and $225 \mathrm{~s}$ ) indicates the CWS in the patient. Bottom, C: Linear relationship between walking speed and 1/time to exhaustion. The Y-intercept corresponds to CWS. 
Physiologic Responses During Different Clinical Exercise Protocols

Besides the incremental shuttle test indicated above, three additional clinical exercise protocols were done in random order on separate days, in the same patients: incremental cycling exercise, 6MWT-90, and stair-climbing test. In all instances, the time elapsed between tests ensured achievement of resting conditions before each protocol.

\section{Measurements}

Lung function at rest ${ }^{21,22}$ was carried out in all patients. A telemetric portable system $\left(\mathrm{K}_{4} \mathrm{~b}^{2}\right.$; Cosmed; Pavona di Albano, Italy) was utilized for on-line exercise measurements in all exercise protocols. ${ }^{13,23}$ A standard incremental shuttle test to volitional fatigue was performed according to the established protocol. ${ }^{24}$ As indicated above, two types of $6 \mathrm{MWT}$ were performed in the present study: $6 \mathrm{MWT}$ carried out in a 10 -m course (6MWT-10) and 6MWT-90; every $30 \mathrm{~s}$, the patients were encouraged by an experienced physiotherapist to continue walking as far as possible. ${ }^{3,25}$ During the stair-climbing test, patients were instructed to climb as far as possible at a brisk pace without the use of railings and to stop at their symptom-limited maximum. ${ }^{26}$ Incremental cycling test was carried out following recommendations previously reported. ${ }^{10}$ Before and at the end of all tests, participants were shown a modified Borg scale printed on a card and asked to indicate their current degree of shortness of breath and leg discomfort on a scale of $0=$ nothing at all, to $10=$ very, very severe. ${ }^{27}$

\section{Statistical Analysis}

Results are expressed as mean $\pm \mathrm{SD}$ or as mean $\pm \mathrm{SEM}$. Comparisons between 6MWT-10 and CWS were done using Student paired $t$ tests and Bland and Altman analyses. ${ }^{28}$ One-way analysis of variance (ANOVA) for repeated measurements was carried out to examine $\dot{\mathrm{V}}_{2}$ profiles at the different exercise protocols. A p value $<0.05$ was considered as statistically significant.

\section{Results}

The study group (mean age, $68 \pm 7$ years; range, 57 to 77 years) showed moderate-to-severe ventilatory dysfunction $\left(\mathrm{FEV}_{1}, 1.63 \pm 0.33 \mathrm{~L} ; 50 \pm 13 \%\right.$ predicted; range, 32 to $67 \%$ predicted). Four patients were classified as Global Initiative for Chronic Obstructive Lung Disease (GOLD) type 2, three patients were GOLD type 3, and one patient was GOLD type $4 .{ }^{8}$ Seven of the eight patients showed air trapping (residual volume, $137 \pm 26 \%$ predicted; range, 80 to $169 \%$ ). On average, they presented moderate hypoxemia $\left(\mathrm{PaO}_{2}, 69 \pm 8 \mathrm{~mm} \mathrm{Hg}\right.$; range, 60 to $82 \mathrm{~mm} \mathrm{Hg}$ ) with a mean $\mathrm{PaCO}_{2}$ of $41 \pm 4 \mathrm{~mm}$ $\mathrm{Hg}$ (range, 38 to $49 \mathrm{~mm} \mathrm{Hg}$ ). Only two patients showed moderate hypercapnia (48 $\mathrm{mm} \mathrm{Hg}$ and 49 $\mathrm{mm} \mathrm{Hg}$, respectively), but none of them showed exercise-induced hypoxemia. Incremental cycling exercise exhibited moderately low peak $\dot{\mathrm{V}}_{2}\left(\dot{\mathrm{VO}}_{2}\right.$ peak): $20.7 \pm 2.4 \mathrm{~mL} / \mathrm{min} / \mathrm{kg}$ (range, 17 to $26 \mathrm{~mL} / \mathrm{min} / \mathrm{kg}$ ).

\section{Assessment of CWS}

Individual relationships between walking speed and time to exhaustion during the four high-intensity, constant-walking speed tests (Fig 2, top, A) showed the expected hyperbolic function indicated in Figure 1, center, $B\left(\mathrm{~L}_{1}\right.$ to $\left.\mathrm{L}_{4}\right)$. All patients presented an excellent linear correlation between constant walking speed expressed as a percentage of peak incremental shuttle and the reciprocal of time to exhaustion (Fig 2, bottom, B). Accordingly, calculated CWS was $1.44 \pm 0.15 \mathrm{~m} / \mathrm{s}$, and the corresponding $\dot{\mathrm{V}}_{2}$ plateau was $1,584 \pm 319 \mathrm{~mL} / \mathrm{min}$. Sustain-

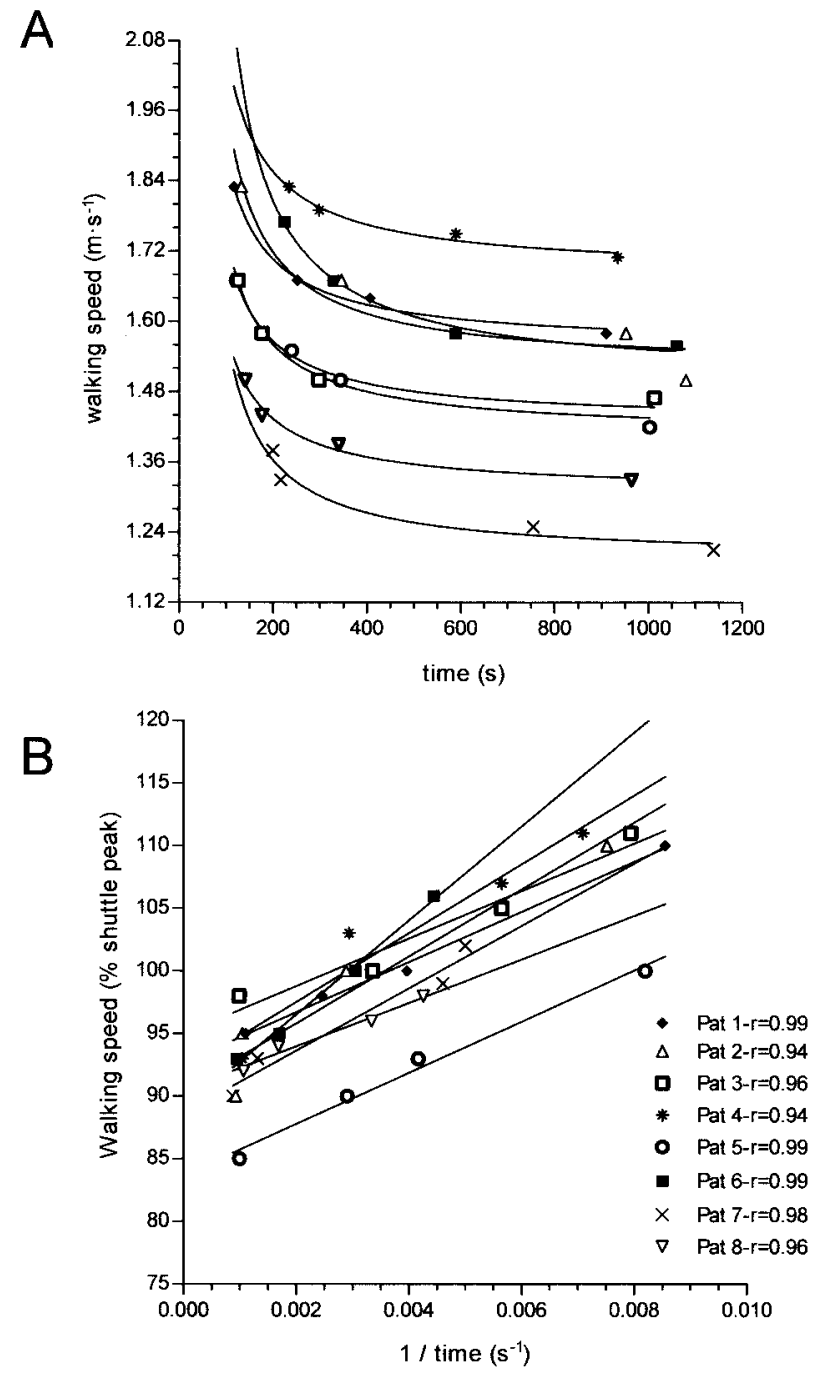

FigURE 2. Individual relationships between walking speed and time to exhaustion for the four high-intensity tests at constant walking speed done in each COPD patient (Pat). Top, A: The hyperbolic relationship between the two variables. Bottom, $B$ : The x-axis shows the reciprocal of time to exhaustion (1/time) that explains the linear transformation of the relationship between the two variables. In this graph, walking speed is expressed as percentage of peak walking speed measured during the incremental shuttle test. Individual coefficients of correlation are indicated. 
ability of walking at CWS for at least 20 min (Fig 1, top, A) was confirmed in all subjects.

Three out of four high-intensity, constant-walking speed tests presented similar $\mathrm{VO}_{2}$ values at exhaustion $\left(\mathrm{L}_{2}, 1,715 \pm 316 \mathrm{~mL} / \mathrm{min} ; \mathrm{L}_{3}, 1,655 \pm 284 \mathrm{~mL} /\right.$ min; and $\left.\mathrm{L}_{4}, 1,660 \pm 239 \mathrm{~mL} / \mathrm{min}\right)$, indicating that maximum walking $\dot{V}_{2}$ (mean value, $1,677 \mathrm{~mL} / \mathrm{min}$ ), calculated as the average of the highest $\dot{\mathrm{O}}_{2}$ for each subject regardless of the speed of the tests $\left(\mathrm{L}_{2}\right.$, $1.52 \pm 0.15 \mathrm{~m} / \mathrm{s} ; \quad \mathrm{L}_{3}, \quad 1.59 \pm 0.15 \mathrm{~m} / \mathrm{s} ;$ and $\mathrm{L}_{4}$, $1.67 \pm 0.17 \mathrm{~m} / \mathrm{s}$ ), was identified in all patients.

\section{Comparisons Between CWS and 6MWT-10}

During the last $3 \mathrm{~min}$ of the $6 \mathrm{MWT}-10, \dot{\mathrm{VO}}_{2}$ showed a plateau in all patients, as reported by others. $^{13}$ Similarly, steady-state conditions $\left(\dot{V}_{\mathrm{O}_{2}}\right.$, minute ventilation $[\dot{\mathrm{V} E}]$, and heart rate $[\mathrm{HR}]$ ) during walking at CWS were also observed in each patient. Consequently, comparisons between walking at CWS and 6MWT-10 were based on mean values of the 3 last min of each test. Figure 3, top, A, displays a strong correlation in $\mathrm{VO}_{2}$ results between CWS $(1,584 \pm 319 \mathrm{~mL} / \mathrm{min})$ and 6MWT-10 (1,605 \pm 304 $\mathrm{mL} / \mathrm{min}$ ), wherein all patients fell close to the identity line $(r=0.93, \mathrm{p}<0.001)$. Likewise, Figure 3, bottom left, $C$, indicates an excellent concordance of $\mathrm{V}_{2}$ between the two exercise tests $(-21 \pm 282$ $\mathrm{mL} / \mathrm{min})$. Figure 3 , top right, $B$, and bottom right, $D$, do not show statistically significant differences in walking speed $(-0.046 \pm 0.14 \mathrm{~m} / \mathrm{s}) \quad$ between
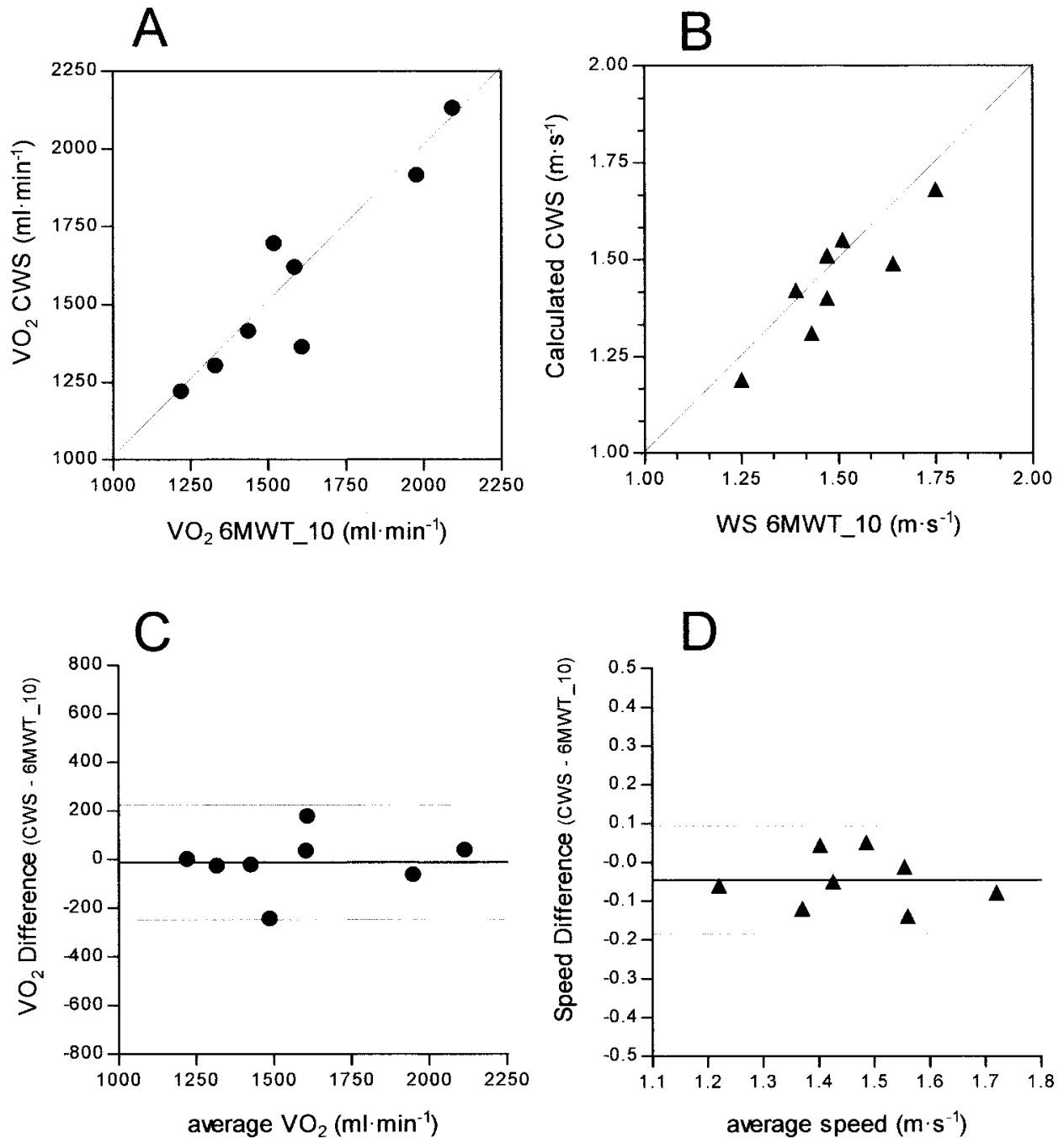

FIgURE 3. Top left, A: Individual relationships between $\dot{\mathrm{V}}_{2}$ during $\mathrm{CWS}$ (y-axis) and $\dot{\mathrm{V}}_{2}$ during 6MWT-10 (x-axis) $[r=0.93, \mathrm{p}<0.001]$. Top right, $B$ : Individual relationships between CWS (y-axis) and walking speed during 6MWT-10 (x-axis) $[r=0.90, \mathrm{p}<0.01]$. Bland and Altman analyses for these two variables $\left(\mathrm{VO}_{2}\right.$ and walking speed) are shown (bottom left, $C$, and bottom right, $D$, respectively). A high concordance between 6MWT-10 and CWS was observed in all instances. 
6MWT-10 (1.49 $\pm 0.16 \mathrm{~m} / \mathrm{s})$ and calculated CWS $(1.44 \pm 0.15 \mathrm{~m} / \mathrm{s})[r=0.90, \mathrm{p}<0.01] . \dot{\mathrm{V}} \mathrm{E}(47 \pm 12$ $\mathrm{L} / \mathrm{min}$ vs $48 \pm 11 \mathrm{~L} / \mathrm{min}, 6 \mathrm{MWT}$ and CWS, respectively); carbon dioxide output $\left(\dot{\mathrm{V}}_{\mathrm{CO}}\right)[1,441 \pm 326$ $\mathrm{mL} / \mathrm{min}$ vs $1,420 \pm 292 \mathrm{~mL} / \mathrm{min}]$; respiratory exchange ratio $(\mathrm{RER})[0.89 \pm 0.1$ vs $0.90 \pm 0.1]$; HR $(130 \pm 18$ beats/min vs $131 \pm 16$ beats/min); and symptom score were also similar between 6MWT-10 and CWS. Borg dyspnea scores at the end of the two tests were $5 \pm 1$ (range, 4 to 8 ) and $6 \pm 2$ (range, 2 to 9 ), respectively; likewise, the Borg leg scores were $4 \pm 2$ (range, 2 to 6 ) and $4 \pm 2$ (range, 1 to 8 ).

\section{Clinical Exercise Protocols}

Figure 4 displays $\dot{\mathrm{V}}_{2}$ profiles during incremental cycling exercise, incremental shuttle test, 6MWT-90, and stair-climbing test measured in the same patients. While 6MWT-90 showed a plateau in $\dot{\mathrm{VO}}_{2}$ after the third minute of the test, ${ }^{13} \mathrm{VO}_{2}$ increased up to the end of the test in the other three exercise protocols. The figure shows an abrupt raise in $\dot{\mathrm{V}}_{2}$ during stair climbing $\left(\dot{\mathrm{V}}_{2}\right.$ at min 1 was $80 \%$ of $\dot{\mathrm{V}}_{2}$ peak) that contrasted with the steady increase of $\dot{\mathrm{VO}}_{2}$ during both incremental cycling and incremental shuttle tests $\left(\mathrm{V}_{2}\right.$ at min 1 were $35 \%$ and $34 \%$ of $\dot{\mathrm{V}}_{2}$ peak, respectively). Detailed information on physiologic responses at the end of each of the four tests is provided in Table 1.

The two types of 6-min walking protocols6MWT-10 and 6MWT-90 — displayed a plateau in $\dot{\mathrm{VO}}_{2}$ after the third minute of the test. 6MWT-10, however, showed higher $\dot{\mathrm{VO}}_{2} \quad(1,605 \pm 304 \mathrm{~mL} / \mathrm{min} \quad$ vs $1,429 \pm 227 \mathrm{~mL} / \mathrm{min} ; \mathrm{p}=0.04)$, lower walking speed $(1.49 \pm 0.16 \mathrm{~m} / \mathrm{s}$ vs $1.63 \pm 0.14 \mathrm{~ms} ; \mathrm{p}=0.0005)$, and shorter walked distance (mean difference, $-54 \pm 1 \mathrm{~m}$; $\mathrm{p}=0.0002)$ than 6MWT-90.

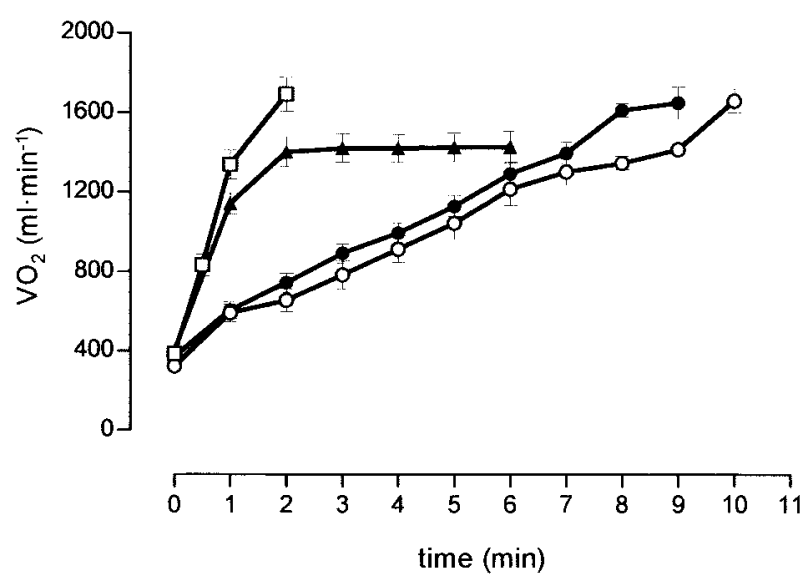

FIgURE 4. Mean $\dot{V}_{2}$ profiles of the eight COPD patients during four different clinical exercise protocols (mean \pm SEM): incremental cycling (open circles), incremental shuttle (solid circles), 6MWT-90 (solid triangles), and stair climbing (open squares).

\section{Discussion}

The current study showed similar physiologic responses between encouraged 6MWT-10 and walking at CWS, which indicates that patients with moderate-to-severe COPD set their walking speed during the test in order to achieve critical $\mathrm{VO}_{2}$. The level of $\dot{\mathrm{V}}_{2}$ achieved at CWS may indicate the integrated response of the systems involved in $\mathrm{O}_{2}$ transport/ $\mathrm{O}_{2}$ utilization that ultimately determine the highest sustainable level of exercise. These results might constitute the underlying explanation for the high prognostic value of the 6MWT.

As described above, the three highest constant walking speeds $\left(\mathrm{L}_{2}\right.$ to $\mathrm{L}_{4}$ in Fig 1 ) showed similar $\dot{\mathrm{VO}}_{2}$ at exhaustion (on average, $1,677 \mathrm{~mL} / \mathrm{min}$ ). Moreover, both stair climbing (1,693 $\pm 256 \mathrm{~mL} /$ $\min )$ and incremental shuttle tests $(1,651 \pm 243$ $\mathrm{mL} / \mathrm{min}$ ) also presented similar peak $\dot{\mathrm{VO}}_{2}$ results (Table 1), thereby further confirming identification of maximum $\mathrm{V}_{2}$ max in all these patients. Maximum $\dot{\mathrm{V}}_{2}$ indicates the maximum capacity of the system for $\mathrm{O}_{2}$ transport $/ \mathrm{O}_{2}$ utilization. It is of most interest that critical $\dot{\mathrm{V}}_{2}\left(\dot{\mathrm{V}}_{2}\right.$ at $\left.\mathrm{CWS}, 1,584 \mathrm{~mL} / \mathrm{min}\right)$ was reported close to $94 \%$ of maximum $\dot{\mathrm{V}}_{2}$ in the present study, as shown by other authors ${ }^{14}$ in COPD patients during incremental cycling.

\section{Does CWS Indicate Critical Power?}

While incremental cycling protocols ${ }^{9,10}$ are designed to facilitate the assessment of the relationship between $\dot{\mathrm{V}}_{2}$ and work rate throughout the test, timed walking tests present important limitations in this regard. It is well accepted that the three most important factors determining energy requirements during encouraged 6MWT are walking speed, ergonomics of the test, and body weight. Since body weight remains unchanged, the other two factors modulate intrasubject variability of energy requirements during the test. The current study provides information on the relationships between these two factors (walking speed and ergonomics) and $\dot{\mathrm{V}}_{2}$.

We observed proportionality between $\dot{\mathrm{VO}}_{2}$ and walking speed during the assessment of CWS, which supports the contention that walking speed was the key determinant of work rate in our patients. This is valid provided that the analysis is done with walking tests carried out in the same corridor length (10-m course in the study), such that similar ergonomics can be reasonably assumed. Moreover, verification of steady-state conditions during exercise at CWS, alike the equivalence between 6MWT-10 and CWS, further indicates the pivotal role of walking speed modulating energy requirements in these patients. The statement requires that self-paced walking speed was constant throughout the time intervals of 
Table 1-Comparison Among Clinical Exercise Protocols*

\begin{tabular}{|c|c|c|c|c|}
\hline Variables & $\begin{array}{c}\text { Stair Climbing } \\
\quad(\text { Peak })\end{array}$ & $\begin{array}{c}\text { Incremental } \\
\text { Cycling } \\
\text { (Peak) }\end{array}$ & $\begin{array}{c}\text { Incremental } \\
\text { Shuttle } \\
\text { (Peak) }\end{array}$ & $\begin{array}{c}\text { 6MWT-90 } \\
\text { (Last } 3 \text { min) }\end{array}$ \\
\hline Time to exhaustion, $\mathrm{s}$ & $88 \pm 37$ & $546 \pm 95$ & $465 \pm 54$ & \\
\hline$\dot{\mathrm{V}} \mathrm{E}, \mathrm{L} / \mathrm{m}$ & $57.7 \pm 14.1$ & $56.0 \pm 8.3$ & $48.2 \pm 10.0$ & $44.2 \pm 10.0 \gamma^{t+}$ \\
\hline$\dot{\mathrm{VO}}_{2}, \mathrm{~mL} / \mathrm{min}$ & $1,693 \pm 256$ & $1,661 \pm 180$ & $1,651 \pm 243$ & $1,429 \pm 227 \nmid t+$ \\
\hline$\dot{\mathrm{V}}_{2}, \mathrm{~mL} / \mathrm{min}$ & $1,749 \pm 359$ & $1,687 \pm 151$ & $1,473 \pm 273 \$$ & $1,273 \pm 220 \nmid+$ \\
\hline RER & $1.03 \pm 0.12$ & $1.02 \pm 0.06$ & $0.89 \pm 0.09 \$ \|$ & $0.89 \pm 0.05 \uparrow^{+}+$ \\
\hline HR, beats/min & $131 \pm 18$ & $133 \pm 15$ & $130 \pm 14$ & $128 \pm 13$ \\
\hline $\mathrm{O}_{2}$ saturation, $\%$ & $91 \pm 5$ & $91 \pm 4$ & $91 \pm 2$ & $90 \pm 5$ \\
\hline Borg score (dyspnea) & $8(3-10)$ & $7(3-9)$ & $5(1-9) \S \|$ & $4(1-7) \nmid+$ \\
\hline Borg score (leg fatigue) & $5(3-8)$ & $6(3-8)$ & $4(1-5) \S$ & $4(1-7) \uparrow$ \\
\hline
\end{tabular}

* Results expressed as mean $\pm \mathrm{SD}$ except for Borg scores, which are expressed as mean (range).

† MWT-90 vs incremental cycling, ANOVA: $\dot{V}_{2}(p<0.01), \dot{V C O}_{2}(p<0.01)$, $\dot{\mathrm{V}}(\mathrm{p}<0.01)$, dyspnea $(\mathrm{p}<0.01), \operatorname{leg}(\mathrm{p}<0.05)$.

$+6 \mathrm{MWT}-90$ vs stair climbing, ANOVA: $\dot{\mathrm{VO}}_{2}(\mathrm{p}<0.005), \dot{\mathrm{V}}_{\mathrm{CO}}(\mathrm{p}<0.01), \dot{\mathrm{VE}}(\mathrm{p}<0.01)$, dyspnea $(\mathrm{p}<0.05)$.

\$Incremental shuttle vs incremental cycling, ANOVA: $\dot{V}_{C_{2}}(\mathrm{p}<0.01)$, RER $(\mathrm{p}<0.01)$, dyspnea $(\mathrm{p}<0.05), \operatorname{leg}(\mathrm{p}<0.05)$.

$\|$ Shuttle vs stair climbing, ANOVA: RER $(\mathrm{p}<0.05)$, dyspnea $(\mathrm{p}<0.05)$.

the encouraged $6 \mathrm{MWT}$, as it was proven in the present study and in previous reports. ${ }^{13,29}$

As described in the "Results" section, 6MWT-10 showed lower efficiency (higher $\mathrm{VO}_{2}$ at lower walking speed) than 6MWT-90, which illustrates that the ergonomics of the test cannot be neglected as a determinant of energy requirements. Clearly, the 10-m course imposed a higher number of turns than 6MWT-90 during the test, which decreased the average speed and increased $\mathrm{VO}_{2}$. The impact of number of turns during the test on the walking distance has been also reported by Sciurba et al. ${ }^{30}$ These results fully support the appropriateness of the current study design that used 6MWT-10 for comparisons with CWS, whereas 6MWT-90 was used to compare with other clinical exercise protocols. It is of note that $\dot{\mathrm{V}}_{2}$ during 6MWT-90 (1,429 $\pm 227 \mathrm{~mL} / \mathrm{min})$ was approximately $85 \%$ of $\dot{\mathrm{V}}_{2}$ max. Further insights on standardization of $6 \mathrm{MWT}$ are clearly beyond the scope of the present investigation.

\section{Exercise Protocols: Physiologic and Clinical Implications}

The most striking feature of encouraged stairs climbing (Fig 4, Table 1) was that approximately $80 \%$ of $\mathrm{V}_{2}$ peak was already achieved within the first minute of the test. This finding rises major concerns on the safety of the test. Moreover, it does not fit with a desirable smooth increase in work rate that may eventually facilitate the analysis of physiologic responses throughout the test. These considerations together with inherent problems for an appropriate standardization of this test limit the usefulness of stairs climbing as a routine clinical test. Incidentally, it has been reported ${ }^{26}$ that nonencouraged stairs climbing protocols may generate a $\dot{\mathrm{VO}}_{2}$ plateau, likely indicating self-adjustment of the patient to achieve sustainable exercise.
Incremental cycling exercise and incremental shuttle test showed similar $\dot{\mathrm{V}}_{2}$ profiles (Fig 4, Table 1). At peak exercise, however, incremental cycling showed higher $\dot{V}_{C O_{2}}$ and higher RER ( $p<0.01$ each) than the incremental shuttle test. Likewise, symptoms score (dyspnea and leg) were also higher $(\mathrm{p}<0.05$ each) during cycling exercise compared to incremental shuttle test. The differences between these two tests could be explained by higher blood lactate levels, hence ventilatory requirements, during incremental cycling compared to timed walking tests. ${ }^{13}$ In the current study, $\dot{V}_{2}$ peak $(1,661 \pm 180 \mathrm{~mL} / \mathrm{min})$ during incremental cycling was similar to $\dot{\mathrm{VO}}_{2}$ at peak incremental shuttle (Table 1), despite the fact that the amount of exercising muscle mass during cycling exercise is significantly smaller than in timed walking tests and the characteristics of the exercise are different. As described above, maximum $\dot{\mathrm{VO}}_{2}$ was observed at exhaustion in most of the exercise protocols carried out in the present study (Fig 1, Table 1), irrespective of the amount of exercising muscle mass. A potential explanation for this finding is that the ceiling of whole-body $\dot{\mathrm{V}}_{2}$ in COPD patients is mainly defined by the degree of pulmonary impairment and not by the amount of exercising muscle mass. The level of exercise is clearly below the vascular constraints to preserve systemic BP observed in healthy man, in whom the amount of exercising muscle mass modulates $\dot{\mathrm{V}}_{2}$ max. $^{31,32}$

The characteristics of the physiologic responses during encouraged 6MWT have been described in the current study and by others. ${ }^{13}$ Standardization and clinical implications of the test are also reported..$^{3,9,10}$ The incremental shuttle test is properly standardized, ${ }^{24}$ and it is widely used for evaluation of patients with chronic respiratory and cardiac disorders. ${ }^{33,34}$

In summary, the current study demonstrates that physiologic responses during 6MWT in patients with 
moderate-to-severe COPD are equivalent to those seen walking at critical power, which corresponds to approximately $90 \%$ of maximum $\mathrm{V}_{2}$ in these patients. The relationship between 6MWT and CWS may likely explain the high predictive value of 6MWT that, together with its simplicity, applicability, and acceptable reproducibility, ${ }^{30,35}$ prompts its recommendation as the most suitable choice for conventional clinical assessment of COPD patients, as part of the multidimensional evaluation of the disease severity. ${ }^{7}$

ACKNOWLEDGMENT: The authors thank Jose Luis Valera, Felip Burgos, Conxi Gistau, and all the technical staff of the lung function laboratory for their skillful support during the study; Rosa Antonucci, visiting scientist from Universita di Roma, La Sapienza (Italy) for her contribution to the study; Elena Gimeno, PT, from EUIF Blanquerna, Universitat Ramon Llull, and Carme Hernandez, coordinator nurse of the Integrated Care Unit, are acknowledged for their outstanding work supervising the logistics of the study. Finally, the authors thank Cosmed (Italy) and Laboratorio Dr Esteve SA for the material support to conduct the study.

\section{REFERENCES}

1 Ferrer M, Alonso A, Morera J, et al. Chronic obstructive pulmonary diseases stage and health-related quality of life: the quality of life of chronic obstructive pulmonary disease study group. Ann Intern Med 1997; 127:1072-1079

2 Jones PW. Health status measurement in chronic obstructive pulmonary disease. Thorax 2001; 56:880-887

3 ATS statement: guidelines for the six-minute walk test. Am J Respir Crit Care Med 2002; 166:111-117

4 Kessler R, Faller M, Fourgaut G, et al. Predictive factors of hospitalization for acute exacerbation in a series of 64 patients with chronic obstructive pulmonary disease. Am J Respir Crit Care Med 1999; 159:158-164

5 Garcia-Aymerich J, Farrero E, Felez MA, et al. Risk factors of readmission to hospital for a COPD exacerbation: a prospective study. Thorax 2003; 58:100-105

6 Gerardi DA, Lovett L, Benoit-Connors ML, et al. Variables related to increased mortality following out-patient pulmonary rehabilitation. Eur Respir J 1996; 9:431-435

7 Celli BR, Cote CG, Marin JM, et al. Combining 6MWD, $\mathrm{FEV}_{1}$, MRC dyspnea and BMI is Better predictor of mortality than $\mathrm{FEV}_{1}[$ abstract]. Am J Respir Crit Care Med 2001; 163(suppl):A504

8 Pauwels RA, Buist AS, Calverley PM, et al. Global strategy for the diagnosis, management, and prevention of chronic obstructive pulmonary disease. NHLBI/WHO Global Initiative for Chronic Obstructive Lung Disease (GOLD) Workshop summary. Am J Respir Crit Care Med 2001; 163:1256-1276

9 ATS/ACCP statement on cardiopulmonary exercise testing. Am J Respir Crit Care Med 2003; 167:211-277

10 European Respiratory Society. Clinical exercise testing with reference to lung diseases: indications, standardization and interpretation strategies. ERS Task Force on Standardization of Clinical Exercise Testing. Eur Respir J 1997; 10:2662-2689

11 Solway S, Brooks D, Lacasse Y, et al. A qualitative systematic overview of the measurement properties of functional walk tests used in the cardiorespiratory domain. Chest 2001; 119:256-270

12 Ambrosino N. Field tests in pulmonary disease. Thorax 1999; 54:191-193

13 Troosters T, Vilaro J, Rabinovich R, et al. Physiological responses to the 6-min walk test in patients with chronic obstructive pulmonary disease. Eur Respir J 2002; 20:564-569

14 Neder JA, Jones PW, Nery LE, et al. Determinants of the exercise endurance capacity in patients with chronic obstructive pulmonary disease: the power-duration relationship. Am J Respir Crit Care Med 2000; 162:497-504

15 Lucas C, Stevenson LW, Johnson W, et al. The 6-min walk and peak oxygen consumption in advanced heart failure: aerobic capacity and survival. Am Heart J 1999; 138:618-624

16 Willenheimer R, Erhardt LR. Value of 6-min-walk test for assessment of severity and prognosis of heart failure. Lancet 2000; 355:515-516

17 Moritani T, Nagata A, deVries HA, et al. Critical power as a measure of physical work capacity and anaerobic threshold. Ergonomics 1981; 24:339-350

18 Revill SM, Morgan MD, Singh SJ, et al. The endurance shuttle walk: a new field test for the assessment of endurance capacity in chronic obstructive pulmonary disease. Thorax 1999; 54:213-222

19 Hill DW. The critical power concept: a review. Sports Med 1993; 16:237-254

20 Gaesser GA, Carnevale TJ, Garfinkel A, et al. Estimation of critical power with nonlinear and linear models. Med Sci Sports Exerc 1995; 27:1430-1438

21 Roca J, Burgos F, Sunyer J, et al. Reference values for forced spirometry. Eur Respir J 1998; 11:1354-1362

22 Roca J, Burgos F, Barbera JA, et al. Prediction equations for plethysmografic lung volumes. Respir Med 1998; 92:454-460

23 Palange P, Forte S, Onorati P, et al. Ventilatory and metabolic adaptations to walking and cycling in patients with COPD. J Appl Physiol 2000; 88:1715-1720

24 Singh SJ, Morgan MDL, Scott S, et al. Development of a shuttle walking test of disability in patients with chronic airflow obstruction. Thorax 1992; 47:1019-1024

25 Guyatt GH, Pugsley SO, Sullivan MJ, et al. Effect of encouragement on walking test performance. Thorax 1984; 39:818822

26 Pollock M, Roa J, Benditt J, et al. Estimation of ventilatory reserve by stair climbing: a study in patients with chronic airflow obstruction. Chest 1993; 104:1378-1383

27 Wilson RC, Jones PW. A comparison of the visual analogue scale and modified Borg scale for the measurement of dyspnea during exercise. Clin Sci 1989; 76:277-282

28 Bland JM, Altman DG. Statistical methods for assessing agreement between two methods of clinical measurement. Lancet 1986; 1:307-310

29 Butland RJ, Pang J, Gross ER, et al. Two-, six-, and 12-minute walking tests in respiratory disease. BMJ (Clin Res Ed) 1982; 284:1607-1608

30 Sciurba F, Criner GJ, Lee SM, et al. Six-minute walk distance in chronic obstructive pulmonary disease: reproducibility and effect of walking course layout and length. Am J Respir Crit Care Med 2003; 167:1522-1527

31 Dempsey JA, Wagner PD. Exercise-induced arterial hypoxemia. J Appl Physiol 1999; 87:1997-2006

32 Rowell LB. Circulatory adjustments to dynamic exercise. In: Rowell LB, ed. Human circulation: regulation during physical stress. New York, NY. Oxford University Press, 1986; 213-256

33 Morales FJ, Montemayor T, Martinez A. Shuttle versus six-minute walk test in the prediction of outcome in chronic heart failure. Int J Cardiol 2000; 76:101-105

34 Keell SD, Chambers JS, Francis DP, et al. Shuttle-walk test to assess chronic heart failure [abstract]. Lancet 1998; 352 : 705

35 Eiser N, Willsher D, Dore CJ. Reliability, repeatability and sensitivity to change of externally and self-paced walking tests in COPD patients. Respir Med 2003; 97:407-414 